\title{
Elevation of the adenylate pool in rat cardiomyocytes by $S$-adenosyl-L-methionine ${ }^{*}$
}

\author{
Ryszard T. Smolenski ${ }^{凶}$ \\ Department of Biochemistry, Medical University of Gdańsk, \\ Dębinki 1, 80-211 Gdańsk, Poland
}

Received: 17 May, 2000; revised: 9 August, 2000; accepted: 17 October, 2000

Key words: cardiomyocytes, ATP, adenylate pool, $S$-adenosyl-L-methionine, adenine, adenosine, ribose

\begin{abstract}
Rapid resynthesis of the adenylate pool in cardiac myocytes is important for recovery of contractility and normal function of regulatory mechanisms in the heart. Adenosine and adenine are thought to be the most effective substrates for nucleotide synthesis, but the possibility of using other compounds has been studied very little in cardiomyocytes. In the present study, the effect of $S$-adenosyl-L-methionine (SAM) on the adenylate pool of isolated cardiomyocytes was investigated and compared to the effect of adenine and adenosine. Adult rat cardiomyocytes were isolated using the collagenase perfusion technique. The cells were incubated in the presence of adenine derivatives for 90 min followed by nucleotide determination by HPLC. The concentrations of adenine nucleotides expressed in $\mathrm{nmol} / \mathrm{mg}$ of cell protein were initially $22.1 \pm 1.4,4.0 \pm 0.3$ and $0.70 \pm 0.08$ for $\mathrm{ATP}, \mathrm{ADP}$ and $\mathrm{AMP}$, respectively $(\mathrm{n}=\mathbf{1 0}$ \pm S.E.M.), and the total adenylate pool was $26.8 \pm 1.6$. In the presence of $1.25 \mathrm{mM} \mathrm{SAM}$ in the medium, the adenylate pool increased by $5.2 \pm 0.4 \mathrm{nmol} / \mathrm{mg}$ of cell protein, but only if $1 \mathrm{mM}$ ribose was additionally present in the medium. No changes were observed with SAM alone. A similar increase (by $4.9 \pm 0.6 \mathrm{nmol} / \mathrm{mg}$ protein) was observed after incubation with $1.25 \mathrm{mM}$ adenine plus $1 \mathrm{mM}$ ribose, but no increase was observed if ribose was omitted. Adenosine at 0.1 or $1.25 \mathrm{mM}$ concentrations also caused an increase in the adenylate pool (by $5.2 \pm 1.0$ and $5.2 \pm 0.9 \mathrm{nmol} / \mathrm{mg}$ protein, respectively), which in contrast to the SAM or adenine was independent of the additional presence of ribose. Thus, $S$-adenosyl-L-methionine could be used as a precursor of the adenylate pool in cardiomyocytes, which is as efficient in increasing the
\end{abstract}

\footnotetext{
This study was supported by the State Committee for Scientific Research (KBN, Poland, ST-41) and the British Heart Foundation (PG/99173).

${ }^{凶}$ Address for correspondence: Dr. Ryszard T. Smolenski, MRS, Heart Science Centre, Imperial College School of Medicine at Harefield Hospital, Harefield, Middx UB9 6JH, U.K.; Tel: (44) 1895828 829; Fax: (44) 1895828 864, e-mail: r.smolenski@ic.ac.uk

Abbreviations: ADRP, adeninediphosphoribose; EHNA, erythro-9-(2-hydroxy-3-nonyl)adenine; MTA, 5'-deoxy-5'-methylthioadenine; PRPP, phosphoribosyl pyrophosphate; SAH, S-adenosylhomocysteine; SAM, S-adenosyl-L-methionine; SAM salt, SAM para-toluenesulphonate salt.
} 
adenylate pool after $90 \mathrm{~min}$ of incubation as adenosine or adenine. Nucleotide synthesis from SAM involves the formation of adenine as an intermediate with its subsequent incorporation by adenine phosphoribosyltransferase.

Myocardial adenylate pool depletion resulting from ischemia may adversely affect metabolic processes and mechanical function in the heart. The association between adenine nucleotide level and contractility has been extensively investigated but a direct link between ATP concentration and the mechanical performance of the heart is still controversial $[1,2]$. However, another consequence of nucleotide pool depletion could be a decreased production of adenosine [3], involved in feedback control mechanisms of coronary flow, adrenergic response, hemostasis and inflammation [4-7] that also may indirectly affect the function of the heart.

Adenine nucleotide synthesis in cardiomyocytes can be accelerated by the supply of adenosine, adenine and, to a limited extent, also by inosine or hypoxanthine [8-10]. Cardiomyocyte nucleotide synthesis from purine bases can be further accelerated by the supply of exogenous ribose [11] because of the relatively low efficiency of the pentose phosphate pathway in the heart and low endogenous ribose production.

Some other derivatives of adenine were also shown to increase the adenylate pool in non cardiac cells. $S$-Adenosyl-L-methionine (SAM), 5'-iodo-5'-deoxyadenosine, neplanocin A or 2 -deoxyadenosine were found to increase the adenylate pool in human erythrocytes [12-14]. Preliminary results indicating that SAM may increase the adenylate pool in cardiac myocytes were included in our preliminary report [15]. However, this effect has not been studied in detail.

The present study was designed to evaluate whether SAM could be used as a precursor of the adenylate pool in cardiomyocytes and to study the mechanism of nucleotide synthesis from SAM. The effect of SAM on the adenine nucleotide pool was compared to that of adenine and adenosine.

\section{MATERIALS AND METHODS}

Isolation of the cells. Cardiac myocytes were isolated from the hearts of Wistar rats weighing 250-300 $\mathrm{g}$ according to a previously described procedure [16]. In brief, animals were anesthetized with diethyl ether, the heart was excised and the aorta cannulated. Perfusion at a flow rate of $10 \mathrm{ml} / \mathrm{min}$ with the low calcium buffer containing $112.6 \mathrm{mM}$ $\mathrm{NaCl}, 15 \mathrm{mM} \mathrm{KCl}, 1.2 \mathrm{mM} \mathrm{KH} \mathrm{PO}_{4}, 1.2 \mathrm{mM}$ $\mathrm{MgSO}_{4}, 10 \mathrm{mM}$ Hepes and $5.5 \mathrm{mM}$ glucose ( $\mathrm{pH}$ 7.3), gassed with $100 \% \mathrm{O}_{2}$ started immediately. The temperature was maintained at $37^{\circ} \mathrm{C}$. After $10 \mathrm{~min}$, perfusion was switched to recirculating mode and the perfusion medium was changed to $20 \mathrm{ml}$ of the above buffer containing $0.1 \%$ collagenase and $0.5 \%$ bovine serum albumin. Calcium readministration started after 15 min of recirculating perfusion and the concentration was raised to $1 \mathrm{mM}$ in three steps with 5 min intervals. After $45 \mathrm{~min}$ of recirculating perfusion, the hearts were removed from the apparatus, the ventricular portion of the heart minced and further incubated for $15 \mathrm{~min}$ at $37^{\circ} \mathrm{C}$ in $10 \mathrm{ml}$ of collagenase solution. After filtration of disaggregated material, cells were purified by sedimentation in $4 \%$ albumin dissolved in phosphate incubation buffer containing: $125 \mathrm{mM} \mathrm{NaCl}, 2.6 \mathrm{mM}$ $\mathrm{KCl}, 1.2 \mathrm{mM} \mathrm{KH} \mathrm{PO}_{4}, 1.2 \mathrm{mM} \mathrm{MgSO}_{4}, 10$ $\mathrm{mM} \mathrm{Na}_{2} \mathrm{HPO}_{4}, 1 \mathrm{mM} \mathrm{CaCl} 2$ and $5.5 \mathrm{mM}$ glucose (pH 7.4). Cells were finally suspended in incubation buffer containing $2 \%$ albumin. Counting the number of rod shaped and rounded cells using light microscopy controlled the quality of the cell preparations. The suspension used for experiments contained $60-70 \%$ of rod shaped cells in a medium containing $1 \mathrm{mM} \mathrm{Ca}{ }^{++}$.

Experimental procedure. Incubation was carried out in plastic Petri dishes. Cell suspension $(0.6 \mathrm{ml})$ was mixed with $0.6 \mathrm{ml}$ of incuba- 
tion buffer containing adenine compounds and/or ribose where indicated. The total cellular protein content was $0.5-1 \mathrm{mg}$ per Petri dish. An inhibitor of adenosine deaminase erythro-9-(2-hydroxy-3-nonyl)adenine (EHNA) - was present in all incubations at $5 \mu \mathrm{M}$ concentration. The incubations were carried out at $37^{\circ} \mathrm{C}$ for $90 \mathrm{~min}$ followed by collection of the cardiomyocyte suspension and separation/extraction according to the procedure described by Geisbuhler et al. [17] with $1.6 \mathrm{ml}$ Eppendorf tubes containing $0.1 \mathrm{ml}$ of $2 \mathrm{M}$ $\mathrm{HClO}_{4}$ covered by a layer of bromododecane $(0.35 \mathrm{ml})$. The myocyte suspension was placed above the bromododecane stratum. This was followed by immediate centrifugation (13000 r.p.m., $4^{\circ} \mathrm{C}, 1 \mathrm{~min}$ ) in an Eppendorf microfuge. Myocytes sedimented into the $\mathrm{HClO}_{4}$ layer, being simultaneously extracted while the medium remained above the bromododecane. After centrifugation, the incubation medium and the bromododecane were removed, $\mathrm{HClO}_{4}$ was diluted with $0.2 \mathrm{ml}$ of distilled water followed by mixing, centrifugation and neutralisation of the collected supernatant with $3 \mathrm{M} \mathrm{K}_{3} \mathrm{PO}_{4}$. The pellet was solubilised in $0.5 \mathrm{M} \mathrm{NaOH}$ and used to deter- by HPLC showed an absence of adenine or adenosine (below 0.1\%) but demonstrated traces of $5^{\prime}$-deoxy-5'-methylthioadenosine (MTA). The sources of chemicals for myocyte isolation, incubation and HPLC were described previously [16, 19].

Statistics. The results are expressed as the mean ( \pm S.E.M.) for different myocyte preparations. Significance of the differences was estimated using one-way analysis of variance followed by Student-Newman-Keuls test with $P<0.05$ considered as a significant difference.

\section{RESULTS}

Table 1 shows the initial content of purine nucleotides in isolated rat cardiomyocytes. No changes of initial values were reported after $90 \mathrm{~min}$ of incubation with the exception of NAD, where some decrease was shown (Fig. 1). During incubation in the presence of adenine derivatives, significant increases in adenine nucleotide content were observed in the presence of SAM with ribose, adenine with ribose and in all adenosine incubations. The nucleotide content after incubation with

Table 1. Initial adenine nucleotide, NAD, NADP and GTP concentrations in the isolated cardiomyocytes.

Values represent the mean \pm S.E.M., $n=10 . T A N=$ total adenine nucleotides $(A T P+A D P+A M P)$.

\begin{tabular}{|c|c|c|c|c|c|c|c|}
\hline & ATP & $\mathrm{ADP}$ & AMP & TAN & GTP & NAD & NADP \\
\hline & \multicolumn{7}{|c|}{$\mathrm{nmol} / \mathrm{mg}$ protein } \\
\hline Mean & 22.1 & 4.0 & 0.70 & 26.8 & 0.62 & 3.80 & 0.11 \\
\hline S.E.M. & \pm 1.4 & \pm 0.3 & \pm 0.08 & \pm 1.6 & \pm 0.04 & \pm 0.33 & \pm 0.02 \\
\hline
\end{tabular}

mine protein content [18]. The nucleotide concentrations were evaluated in cell extracts using a previously described reversed-phase HPLC method [19, 20] and are expressed in $\mathrm{nmol} / \mathrm{mg}$ of cell protein.

Reagents. SAM (para-toluenesulphonate salt), adenine, adenosine, $5^{\prime}$-deoxy-5' $5^{\prime}$-methylthioadenosine and ribose were from Sigma (St. Louis, U.S.A.). Analysis of SAM for purity
MTA and ribose also showed a positive trend but this difference did not reach statistical significance.

The GTP concentration was $0.62 \pm 0.04$ $\mathrm{nmol} / \mathrm{mg}$ protein initially and did not change significantly under various incubation conditions (Fig. 1B). The initial NAD content was $3.80 \pm 0.33 \mathrm{nmol} / \mathrm{mg}$ protein and a slight decrease was observed during $90 \mathrm{~min}$ of incuba- 

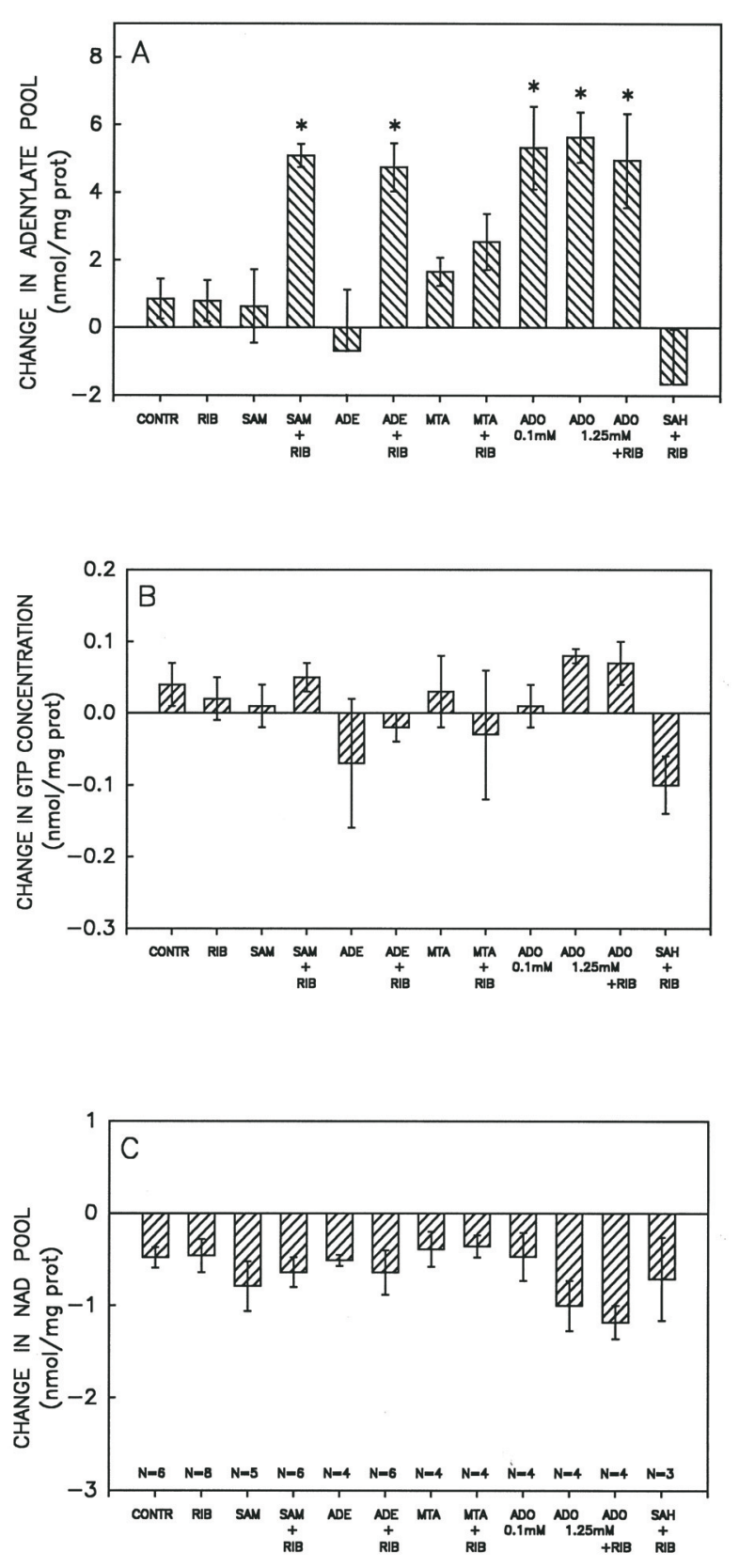

Figure 1. Changes in (A) adenylate pool (ATP+ ADP+AMP), (B) GTP concentration and (C) NAD concentration in rat cardiomyocytes incubated for $90 \mathrm{~min}$ at $37^{\circ} \mathrm{C}$ in medium containing $125 \mathrm{mM}$ $\mathrm{NaCl}, 2.6 \mathrm{mM} \mathrm{KCl}, 1.2 \mathrm{mM} \mathrm{KH}_{2} \mathrm{PO}_{4}, 1.2 \mathrm{mM}$ $\mathrm{MgSO}_{4}, 10 \mathrm{mM} \mathrm{NaH} \mathrm{PO}_{4}, 1 \mathrm{mM} \mathrm{CaCl} 2,5.5 \mathrm{mM}$ glucose and $5 \mu \mathrm{M}$ EHNA (pH 7.4).

The following compounds were present where indicated SAM, $S$-adenosyl-L-methionine (1.25 mM), ADE, adenine (1.25 mM), MTA, 5'-deoxy-5'-methylthioadenosine $(0.1 \mathrm{mM})$, adenosine, $\mathrm{ADO}(0.1$ or $1.25 \mathrm{mM})$, SAH, $S$-adenosylhomocysteine (1.25 mM), RIB, ribose (1 $\mathrm{mM})$. All values in (A), (B) and (C) represent the mean \pm S.E.M. of $\mathrm{N}$ given in $(\mathrm{C})$. ${ }^{*} P<0.05$ vs CONTR, RIB, SAM, ADE, SAH+RIB. tion (Fig. 1C) but the degree of this decrease was not significantly different among the groups.

\section{DISCUSSION}

Demonstration that $S$-adenosyl-L-methionine (SAM) is capable of increasing the adenine nucleotide content in cardiac myocytes is the most interesting observation of this study. This increase in the adenylate pool was observed only if ribose was additionally present in the incubation medium, suggesting that adenine is an intermediate of this process and that it involves the adenine phosphoribosyltransferase reaction.

Nucleotide synthesis from SAM was demonstrated earlier but was adequately studied only in human erythrocytes [12, 21]. These studies provided evidence that the mechanism of increase in the adenylate pool in the presence of SAM is independent of adenosine kinase activity but as intact adenine phosphoribosyltransferase pathway is obligatory. This, in turn, suggested that adenine has to be as intermediate of this process. In the present study with rat cardiomyocytes, a similar pathway of SAM conversion to adenine nucleotides can be proposed. This conclusion is supported by the observation that the effect of SAM was totally dependent on the additional presence of ribose, which is the precursor of PRPP, a co-substrate of the adenine phosphoribosyltransferase reaction. However, the mechanism of SAM conversion to adenine cannot be established with certainty according to present or earlier studies. Contamination of SAM preparation by adenine can be excluded because of the reagent purity control with HPLC. Slight contamination of SAM by 5'-deoxy-5'-methylthioadenosine (MTA), which is a potential source of adenine via the MTA phosphorylase reaction [22], is unlikely to be the cause of the increase in ATP observed for two reasons. At first, the experiment with MTA demonstrated a less significant effect on 
the adenylate pool at concentrations far exceeding the MTA content during incubation with SAM. Secondly, some experiments were performed with a batch of SAM reagent almost free from MTA but as increase in the ATP and nucleotide pool was still observed. A conversion of SAM to the adenine during the incubation must thus be an obligatory step but the details of this process remains to be resolved.

Considering the possible mechanisms of this conversion, both non-enzymatic and enzymatic processes have to be taken into account. SAM is an unstable compound that can generate MTA and adenine at $37^{\circ} \mathrm{C}[23,24]$ but also may be involved in extracellular enzymatic reactions [25]. However, extracellular transmethylations that release $S$-adenosylhomocysteine (SAH) from SAM can be excluded because extracellular SAH did not exert any effect on the adenylate pool (Fig. 1). This also confirms that SAH cannot be transported across the cardiac cell membrane even after prolonged incubation, as in this study, since inward transport of SAH would lead to an elevation of the nucleotide pool following the breakdown of SAH to adenosine in the $\mathrm{SAH}$ hydrolase pathway [16].

In contrast to SAH, transmembrane SAM transport has been demonstrated in human erythrocytes [26]. An active specific inward transport of SAM was also demonstrated in lower organisms [27]. The possibility that SAM could be transported into the cell as an intact molecule in mammals is suggested by its strong cytoprotective and anticancer effects in the liver [28-31] which depend on methyl group donor function of SAM. Interestingly, an improved protection of the nucleotide pool against ethanol toxic effect was found to be associated with SAM treatment in hepatocytes [32]. Intracellular uptake is an essential step of any further conversion of SAM within the cell, including reactions leading to the release of intracellular SAH, MTA or adenine which then could be used for adenylate synthesis.
The influence of adenine on the adenylate pool was similar to the effect of SAM, both with regard to the magnitude of the nucleotide pool elevation and dependence of this effect on the additional presence of ribose. This provides further support that both processes must involve some similar steps. This is surprising considering how much faster adenosine could be incorporated into the cardiomyocyte nucleotide pool than adenine, even in the presence of ribose [8, 9]. A possible explanation, although not very likely at our low 0.1 $\mathrm{mM}$ concentration, is substrate inhibition of adenosine kinase [10, 33]. It is also possible that nucleotide synthesis from both nucleoside and base precursor is feedback inhibited when the nucleotide pool reaches a certain level. It was the aim of this study to establish the long-term effect of nucleotide precursors on the nucleotide pool in cardiac cells rather than measuring initial rates of incorporation. This was the reason why we chose to incubate cells for a prolonged time at relatively high nucleotide precursor concentrations. Establishing exact time courses and exact concentration dependences of nucleotide pool changes following incubation with different nucleotide substrates would be an important extension of this research.

There was a slight decrease in NAD content after 90 min of incubation, which is probably a consequence of some loss in cell membrane integrity or a shift into NADH following acid extraction that could be detected as adenine diphosphoribose (ADPR). In fact, some increase in ADPR was observed following incubation (data not shown). However, there were no significant differences in NAD concentration change or ADPR accumulation under any of the incubation conditions. The adenine compounds used here thus selectively affected the adenylate pool.

This study demonstrated that SAM could be a substrate of cardiomyocyte adenylate pool synthesis. The effect of SAM was dependent on the presence of ribose, suggesting the formation of adenine as an intermediate metabo- 
lite but identification of the detailed pathway of SAM conversion to adenine and then to nucleotides requires further studies. Whatever is the mechanism, SAM and ribose administration appears to be a possible treatment to restore the adenine nucleotide pool in the heart after ischemia during cardiac surgery or in the course of ischemic heart disease. A decreased nucleotide pool under such conditions is an important problem widely discussed [34]. It is important that SAM is used widely clinically not only as hepatic drug [35, 36] but also in the treatment of psychological and neurological disorders [37] without any apparent deleterious effects. SAM could be superior to adenine since there is some risk of kidney stones associated with the administration of adenine [38, 39]. Evaluation of the cardioprotective properties of SAM administered together with ribose is certainly worthwhile further investigation.

\section{R E F E R E N C E S}

1. Achterberg, P.W. (1988) Adenine nucleotides, purine metabolism and myocardial function; in Myocardial Energy Metabolism (de Jong, J.W., ed.) pp. 45-52, Nijhof Publs, Dordrecht, Boston, Lancaster.

2. Kupriyanov, V.V., Lakomkin, V.L., Kapelko, V.I., Steinschneider, A.Y., Ruuge, E.K. \& Saks, V.A. (1987) Dissociation of adenosine triphosphate levels and contractile function in isovolumetric hearts perfused with 2-deoxyglucose. J. Mol. Cell. Cardiol. 19, 729-740.

3. Smolenski, R.T., Simmonds, H.A. \& Chambers, D.J. (1997) Exogenous adenosine supplied transiently during reperfusion ameliorates depressed endogenous adenosine production in the post-ischemic rat heart. J. Mol. Cell. Cardiol. 29, 333-346.

4. Berne, R.M. (1980) The role of adenosine in the regulation of coronary blood flow. Circ. Res. 47, 807-813.
5. Schrader, J., Baumann, G. \& Gerlach, E. (1977) Adenosine as inhibitor of myocardial effects of catecholamines. Pflugers Arch. 372, $29-35$.

6. Mills, D.C.B., MacFarlane, D.E., Lemmex, B.W.G. \& Haslam, R.J. (1983) Receptors for nucleosides and nucleotides on blood platelets; in Regulatory Functions of Adenosine (Berne, R.M. et al., eds.) pp. 277-289. Nijhof Publs, The Hague, Boston, London.

7. Cronstein, B.N., Levin, R.I., Philips, M., Hirschhorn, R., Abramson, S.B. \& Weissmann, G. (1992) Neutrophil adherence to endothelium is enhanced via adenosine A1 receptors and inhibited via adenosine A2 receptors. J. Immunol. 148, 2201-2206.

8. Brown, A.K., Raeside, D.L., Bowditch, J. \& Dow, J.W. (1985) Metabolism and salvage of adenine and hypoxanthine by myocytes isolated from mature rat heart. Biochim. Biophys. Acta 845, 469-476.

9. Bowditch, J., Brown, A.K. \& Dow, J.W. (1985) Accumulation and salvage of adenosine and inosine by isolated mature cardiac myocytes. Biochim. Biophys. Acta 844, 119-128.

10. Dow, J.W., Bowditch, J., Nigdikar, S.V. \& Brown, A.K. (1987) Salvage mechanisms for regeneration of adenosine triphosphate in rat cardiac myocytes. Cardiovasc. Res. 21, 188196.

11. Zimmer, H.G. \& Ibel, H. (1984) Ribose accelerates the repletion of the ATP pool during recovery from reversible ischemia of the rat myocardium. J. Mol. Cell. Cardiol. 16, 863866.

12. Montero, C., Smolenski, R.T., Duley, J.A. \& Simmonds, H.A. (1990) $S$-adenosylmethionine increases erythrocyte ATP in vitro by a route independent of adenosine kinase. Biochem. Pharmacol. 40, 2617-2623.

13. Smolenski, R.T., Fabianowska-Majewska, K., Montero, C., Duley, J.A., Fairbanks, Marlewski, M. \& Simmonds, H.A. (1992) A novel 
route of ATP synthesis. Biochem. Pharmacol. 43, 2053-2057.

14. Smolenski, R.T., Montero, C., Duley, J. \& Simmonds, H.A. (1991) Effects of adenosine analogues on ATP concentration in human erythrocytes. Further evidence for a route independent of adenosine kinase. Biochem. Pharmacol. 42, 1767-1773.

15. Montero, C., Smolenski, R.T., Duley, J.A. \& Simmonds, H.A. (1991) An alternative pathway of adenylate and ATP synthesis. Adv. Exp. Med. Biol. 309A, 285-288.

16. Smolenski, R.T., Schrader, J., de Groot, H. \& Deussen, A. (1991) Oxygen partial pressure and free intracellular adenosine of isolated cardiomyocytes. Am. J. Physiol. 260, C708C714.

17. Geisbuhler, T., Altschuld, R.A., Trewyn, R.W., Ansel, A.Z., Lamka, K. \& Brierley, G.P. (1984) Adenine nucleotide metabolism and compartmentalization in isolated adult rat heart cells. Circ. Res. 54, 536-546.

18. Lowry, O.H., Rosebrough, N.J., Farr, A.L. \& Randall, R.J. (1951) Protein measurement with the Folin phenol reagent. J. Biol. Chem. 193, 265-275.

19. Smolenski, R.T., Lachno, D.R., Ledingham, S.J.M. \& Yacoub, M.H. (1990) Determination of sixteen nucleotides, nucleosides and bases using high-performance liquid chromatography and its application to the study of purine metabolism in hearts for transplantation. $J$. Chromatogr. 527, 414-420.

20.Smolenski, R.T., Skladanowski, A.C., Swierczynski, J., Perko, M., Narkiewicz, M. \& Zydowo, M.M. (1993) Changes of nucleotide content in human and rat heart during cardiac surgery and ischemia. Acta Biochim. Polon. 40, 531-538.

21. Maeda, N., Kon, K., Sekiya, M. \& Shiga, T. (1986) Increase of ATP level in human erythrocytes induced by $S$-adenosyl-L-methionine. Biochem. Pharmacol. 35, 625-629.
22. Sahota, A., Webster, D.R., Potter, C.F., Simmonds, H.A., Rodgers, V.A. \& Gibson, T. (1983) Methylthioadenosine phosphorylase activity in human erythrocytes. Clin. Chim. Acta 128, 283-290.

23. Hoffman, J.L. (1986) Chromatographic analysis of the chiral and covalent instability of $S$-adenosyl-L-methionine. Biochemistry 25, 4444-4449.

24. Revelle, L.K., d'Avignon, D.A., Reepmeyer, J.C. \& Zerfing, R.C. (1995) Stability-indicating proton nuclear magnetic resonance spectroscopic method for determination of $S$-adenosyl-L-methionine in tablets. J. AOAC. Int. 78, 353-358.

25. Barber, J.R., Morimoto, B.H., Brunauer, L.S. \& Clarke, S. (1986) Metabolism of S-adenosyl-L-methionine in intact human erythrocytes. Biochim. Biophys. Acta, 886, 361-372.

26. Stramentinoli, G., Pezzoli, C. \& Kienle, M.G. (1978) Uptake of $S$-adenosyl-L-methionine by rabbit erythrocytes. Biochem. Pharmacol. 27, 1427-1430.

27. Avila, J.L. \& Polegre, M.A. (1993) Uptake and metabolism of $S$-adenosyl-L-methionine by Leishmania mexicana and Leishmania braziliensis promastigotes. Mol. Biochem. Parasitol. 58, $123-134$.

28. Gerbracht, U., Eigenbrodt, E., Simile, M.M., Pascale, R.M., Gaspa, L., Daino, L., Seddaiu, M.A., De Miglio, M.R., Nufris, A. \& Feo, F. (1993) Effect of $S$-adenosyl-L-methionine on the development of preneoplastic foci and the activity of some carbohydrate metabolizing enzymes in the liver, during experimental hepatocarcinogenesis. Anticancer Res. 13, 1965-1972.

29. Lieber, C.S. (1994) Hepatic and metabolic effects of ethanol: Pathogenesis and prevention. Ann. Med. 26, 325-330.

30. Muriel, P., Suarez, O.R., Gonzalez, P. \& Zuniga, L. (1994) Protective effect of S-adenosyl-L-methionine on liver damage induced by 
biliary obstruction in rats: A histological, ultrastructural and biochemical approach. $J$. Hepatol. 21, 95-102.

31. Alvaro, D., Gigliozzi, A., Piat, C., Carli, L., Bini, A., La Rosa, T., Furfaro, S. \& Capocaccia, L. (1995) Effect of $S$-adenosyl-L-methionine on ethanol cholestasis and hepatotoxicity in isolated perfused rat liver. Dig. Dis. Sci. 40, 1592-1600.

32. Garcia Ruiz, C., Morales, A., Colell, A., Ballesta, A., Rodes, J., Kaplowitz, N. \& Fernandez Checa, J.C. (1995) Feeding $S$-adenosyl-L-methionine attenuates both ethanol-induced depletion of mitochondrial glutathione and mitochondrial dysfunction in periportal and perivenous rat hepatocytes. Hepatology 21, 207-214.

33. Arch, J.R.S. \& Newsholme, E.A. (1978) Activities and some properties of $5^{\prime}$-nucleotidase, adenosine kinase and adenosine deaminase in tissues from vertebrates and invertebrates in relation to the control of the concentration and the physiological role of adenosine. Biochem. J. 174, 965-977.

34. Smolenski, R.T. \& Yacoub, M.H. (1996) Purine metabolism in human cardiomyocytes and endothelium - implications for protection of the heart during cardiac surgery; in Purines and Myocardial Protection (Abd-Elfattah, A.S.A. et al., eds.) pp. 55-80, Kluwer Academic Publishers, Norwell.

35. Lieber, C.S. (1999) Role of S-adenosyl-L-methionine in the treatment of liver diseases. $J$. Hepatol. 30, 1155-1159.

36. Net, M., Garcia-Valdecasas, J.C., Deulofeu, R., Gonzalez, X., Palacin, J., Almenara, R., Valero, R., Lopez-Boado, M., Angas, J., Elena, M., Ballesta, A.M. \& Visa, J. (1999) S-Adenosyl-L-methionine effect on hepatic allografts procured from non-heart-beating donor pigs. Transplant. Proc. 31, 1063-1064.

37. Benson, R., Crowell, B., Hill, B., Doonquah, K. \& Charlton, C. (1993) The effects of L-dopa on the activity of methionine adenosyltransferase relevance to L-dopa therapy and tolerance. Neurochem. Res. 18, 325-330.

38. Simmonds, H.A., Van Acker, K.J., Cameron, J.S. \& Snedden, W. (1976) The identification of 2,8-dihydroxyadenine, a new component of urinary stones. Biochem. J. 157, 485-487.

39. Cameron, J.S., Simmonds, H.A., Cadenhead, A. \& Farebrother, D. (1977) Metabolism of intravenous adenine in the pig. Adv. Exp. Med. Biol. 76A, 196-205. 